\title{
Regional Gastrointestinal Transit Times in Patients With Carcinoid Diarrhea: Assessment With the Novel 3D-Transit System
}

\author{
Tine Gregersen, ${ }^{1 *}$ Anne-Mette Haase, ${ }^{1}$ Vincent Schlageter, ${ }^{2}$ Henning Gronbaek, ${ }^{1}$ and Klaus Krogh ${ }^{1}$ \\ ${ }^{1}$ Neurogastroenterology Unit, Department of Hepatology and Gastroenterology, Aarhus University Hospital, Aarhus, Denmark; and ${ }^{2}$ Motilis \\ Medica SA, Lausanne, Switzerland
}

\begin{abstract}
Background/Aims
The paucity of knowledge regarding gastrointestinal motility in patients with neuroendocrine tumors and carcinoid diarrhea restricts targeted treatment. 3D-Transit is a novel, minimally invasive, ambulatory method for description of gastrointestinal motility. The system has not yet been evaluated in any group of patients. We aimed to test the performance of 3D-Transit in patients with carcinoid diarrhea and to compare the patients' regional gastrointestinal transit times (GITT) and colonic motility patterns with those of healthy subjects.
\end{abstract}

\section{Methods}

Fifteen healthy volunteers and seven patients with neuroendocrine tumor and at least 3 bowel movements per day were investigated with 3D-Transit and standard radiopaque markers.

\section{Results}

Total GITT assessed with 3D-Transit and radiopaque markers were well correlated (Spearman's rho $=0.64, P=0.002$ ). Median total GITT was 12.5 (range: $8.5-47.2$ ) hours in patients versus 25.1 (range: $13.1-142.3$ ) hours in healthy $(P=0.007)$. There was no difference in gastric emptying $(P=0.778)$. Median small intestinal transit time was 3.8 (range: $1.4-5.5)$ hours in patients versus 4.4 (range: $1.8-7.2$ ) hours in healthy subjects $(P=0.044)$. Median colorectal transit time was 5.2 (range: $2.9-40.1)$ hours in patients versus 18.1 (range: $5.0-134.0$ ) hours in healthy subjects $(P=0.012)$. Median frequency of pansegmental colonic movements was 0.45 (range: $0.03-1.02$ ) per hour in patients and 0.07 (range: $0-0.61$ ) per hour in healthy subjects $(P$ $=0.045)$.

\section{Conclusions}

Three-dimensional Transit allows assessment of regional GITT in patients with diarrhea. Patients with carcinoid diarrhea have

Received: February 28, 2015 Revised: April 4, 2015 Accepted: April 5, 2015

(c) This is an Open Access article distributed under the terms of the Creative Commons Attribution Non-Commercial License (http://creativecommons. org/licenses/by-nc/4.0) which permits unrestricted non-commercial use, distribution, and reproduction in any medium, provided the original work is properly cited.

*Correspondence: Tine Gregersen, MD

Aarhus University Hospital, Norrebrogade 44, Building 1A, Basement, DK-8000 Aarhus C, Denmark

Tel: +45-2233-4161, Fax: +45-7846-2860, E-mail: tg@clin.au.dk

Financial support: The study was supported by Novartis (unrestricted research grant). Tine Gregersen received a research grant from Aarhus University, and Henning Gronbæk was supported by the NOVO Nordisk Foundation (clinical research grant).

Conflicts of interest: Vincent Schlageter is co-owner of Motilis Medica SA.

Author contributions: Tine Gregersen, study design, data collection, interpretation, and analysis, preparation of manuscript, critical review, and guarantor of the article; Anne-Mette Haase, data interpretation and analysis, and critical review; Vincent Schlageter, technical support and technical review; Henning Gronbaek, study design, data collection, and critical review; Klaus Krogh, study design, data collection, preparation of manuscript, and critical review. All authors have made substantial contribution to the study. All authors have read and approved the final manuscript.

ORCID: Tine Gregersen, http://orcid.org/0000-0002-4657-3918; Anne-Mette Haase, http://orcid.orc/0000-0001-7093-8350; Vincent Schlageter, http://orcid.org/0000-0001-6305-5103; Henning Gronbaek, http://orcid.org/0000-0003-0745-3259; Klaus Krogh, http://orcid.org/0000-0001-9168-2403. 
faster than normal gastrointestinal transit due to faster small intestinal and colorectal transit times. The latter is caused by an increased frequency of pansegmental colonic movements.

\section{(J Neurogastroenterol Motil 2015;21:423-432)}

\section{Key Words}

Capsules; Carcinoid tumor; Diarrhea; Gastrointestinal motility

\section{Introduction}

Diarrhea has a major impact on the health related quality of life in patients with the carcinoid syndrome caused by neuroendocrine tumors (NETs). ${ }^{1}$ The diarrhea usually occurs when the primary tumor has metastasized to the liver or the retroperitoneal space, and tumor products are able to escape hepatic first-pass metabolism and reach the systemic circulation. ${ }^{2}$ Several NETs secrete amines and peptide hormones that can affect gastrointestinal (GI) motility. Among these, serotonin (5-HT) is considered the most important. ${ }^{3}$ Intravenously administered 5-HT enhances phase III migrating myoelectric complexes of the stomach and small intestine and doubles the migrating velocity in healthy subjects. ${ }^{4}$ In animal studies, 5 -HT stimulates the amplitude of contractions in the gastric antrum, duodenum, and ileum and also enhances $5-\mathrm{HT}_{4}$ receptor-mediated colonic secretion. ${ }^{5,6}$ In rats, intravenously administered 5-HT shortens the interval between migrating myoelectric complexes in a dose-dependent fashion. ${ }^{7}$ Intravenously administered citalopram (a selective 5-HT reuptake inhibitor) increases the frequency of high-amplitude propagated contractions in human colon. ${ }^{8}$

The standard treatment of carcinoid diarrhea is somatostatin analogues (SA), ${ }^{9-13}$ which reduce diarrhea in 33-90\% of patients. ${ }^{2,14,15}$ However, side effects to treatment with SA also include diarrhea as reported in up to $26 \%$ of patients. ${ }^{16}$ Only very few studies exist on GI motility patterns and transit times in patients with carcinoid diarrhea. ${ }^{15,17}$ With scintigraphy, von der Ohe et $\mathrm{al}^{17}$ found that patients with NET had faster than normal small intestinal and colonic transit times. In a previous study with a system based on the tracking of a permanent magnetic pill (MTS-1), we found that patients with NET had normal gastric emptying but faster than normal small intestinal transit. ${ }^{15}$ There were, however, major limitations with all studies based on MTS-1, including our earlier study in patients with NET, because the subjects had to be immobile and restricted to a non-magnetic bed. This limited the study period to 6 hours and prohibited examination of colorectal transit patterns.

3D-Transit ${ }^{\mathrm{TM}}$ (Motilis Medica SA, Lausanne, Switzerland) is a new, promising, minimally invasive method for assessment of regional transit times and motility patterns throughout the GI tract. As the system is based on electromagnets, it allows ambulatory study under near normal physiological circumstances. In a pilot study among healthy subjects, 3D-Transit was well-tolerated and provided valid information about GI transit times with only minimal data loss. ${ }^{18}$ However, no data on the use of 3D-Transit has yet been published in any patient group.

We hypothesized that 3D-Transit would be safe and well tolerated in this group of patients and that total GI transit times assessed with 3D-Transit are well correlated to those determined with standard radiopaque markers. We further hypothesized that patients with NET have faster than normal small intestinal, colorectal, and total GI transit times.

As such the aims of this study were dual (1) to evaluate the ambulatory use of 3D-Transit in a group of patients with severe diarrhea and (2) to describe regional GI transit times and colonic motility patterns in patients with diarrhea as part of the carcinoid syndrome.

\section{Materials and Methods}

\section{Patients and Healthy Volunteers}

Seven patients ( 6 males; median age 69 years [range, 63-79 years]) with pathology verified metastatic NET and a minimum of 3 bowel movements per day were compared with 15 healthy subjects ( 5 males; median age 55 years [range, 47-79 years]) with normal bowel function and no history of GI surgery or current use of medication affecting GI function. Patients were included from the ENETS Center of Excellence, Department of Hepatology and Gastroenterology, Aarhus University Hospital, Denmark. Demographic and clinical characteristics of patients are listed in Table 1.Three were treatment-naïve and four had not received treatment with long acting SA for a minimum of 6 
Table 1. Patient Demographics and Clinical Characteristics

\begin{tabular}{|c|c|c|c|c|c|c|c|c|}
\hline No & Gender & Age & $\begin{array}{l}\text { Primary } \\
\text { tumor }\end{array}$ & Metastases & $\begin{array}{c}\text { Bowel } \\
\text { movements } \\
\text { per day }\end{array}$ & $\begin{array}{c}\begin{array}{c}\text { Serotonin } \\
(\mathrm{nmol} / \mathrm{L})\end{array} \\
\text { Normal }<30\end{array}$ & $\begin{array}{c}\text { U5-HIAA } \\
(\mu \mathrm{mol} / 24 \mathrm{hr}) \\
\text { Normal }<40\end{array}$ & $\begin{array}{c}\mathrm{CgA} \\
(\mathrm{pmol} / \mathrm{L}) \\
\text { Normal }<130\end{array}$ \\
\hline 1 & Female & 64 & Small intestine & Liver & 4 & 22 & 34 & 260 \\
\hline 2 & Male & 67 & Pancreas & Liver & 3 & $<10$ & 79 & 148 \\
\hline 3 & Male & 72 & Small intestine & $\begin{array}{l}\text { Liver } \\
\text { Mesentery } \\
\text { Retroperitoneal glands }\end{array}$ & 6 & 167 & 1712 & 9200 \\
\hline 4 & Male & 69 & Small intestine & $\begin{array}{l}\text { Liver } \\
\text { Bones }\end{array}$ & 6 & 514 & 1574 & 7750 \\
\hline 5 & Male & 79 & Small intestine & $\begin{array}{l}\text { Liver } \\
\text { Mesentery } \\
\text { Retroperitoneal glands }\end{array}$ & 8 & 47 & Missing & 18400 \\
\hline 6 & Male & 63 & Small intestine & $\begin{array}{l}\text { Liver } \\
\text { Bones } \\
\text { Retroperitoneal glands }\end{array}$ & 5 & 7020 & 1399 & 9120 \\
\hline 7 & Male & 70 & Small intestine & $\begin{array}{l}\text { Liver } \\
\text { Bones } \\
\text { Lung }\end{array}$ & 6 & 63 & 289 & 15100 \\
\hline
\end{tabular}

U5-HIAA, Urine 5-hydroxyindoleacetic acid; CgA, chromogranin A.

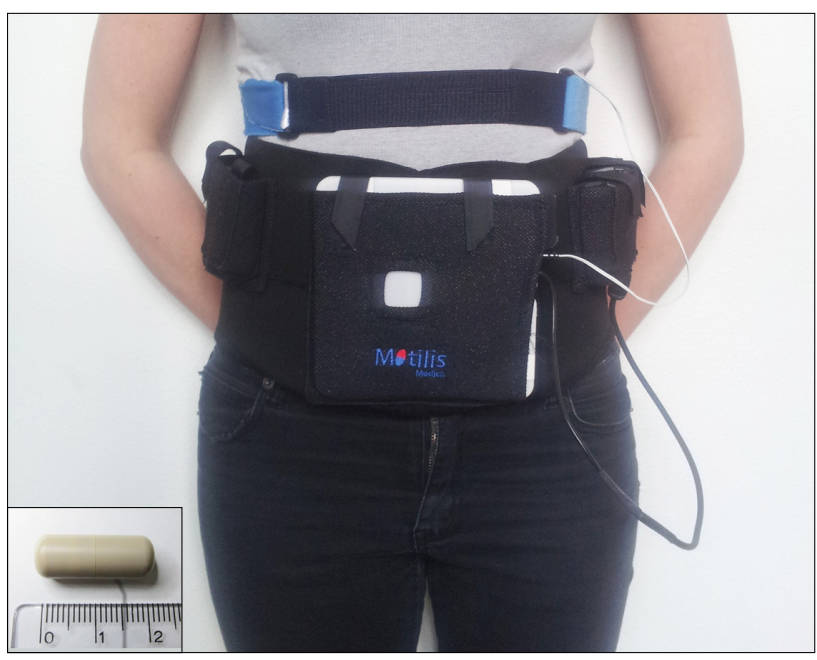

Figure 1. 3D-Transit system. Electromagnetic signals from the ingestible capsule (inset) are registered by sensors in the detector plate. The detector plate and its battery are worn in an abdominal belt. A respiration belt is placed above the detector to remove respiration artefacts. Data are stored on a SD card in the detector.

weeks. All other medications affecting GI motility were discontinued during the study. In one of the patients, the primary tumor had been resected with only $23 \mathrm{~cm}$ of the small intestine 50 $\mathrm{cm}$ proximal to the ileocecal valve. The rest had not undergone bowel resection. The patients with NET were generally older than the healthy subjects from our previous method study. ${ }^{18}$

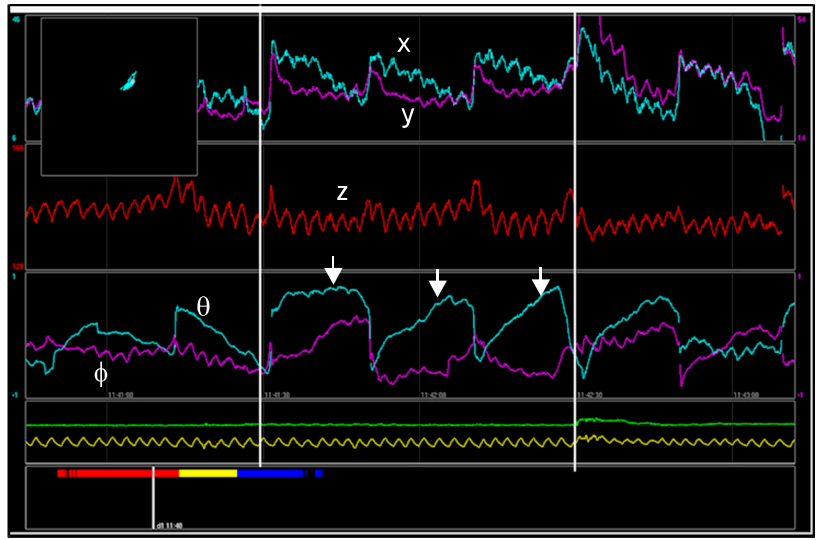

Figure 2. Graphs depicting data from the ingested capsule using dedicated software. The position (x,y, and $z$ ) and orientation $(\Phi$ and $\theta$ ) of the capsule are displayed. The arrows indicate the contraction frequency, in this case the 3 contractions per minute characteristic of the stomach. Yellow line displays respiration. In the upper left corner position of the capsule is monitored.

However, the oldest 4 subjects from our previous study were included in the present study along with another 11 healthy subjects of appropriate age.

The study was conducted in accordance with the Declaration of Helsinki, approved by the local scientific ethics committee and the Danish Medical Agency (Eudract No. 2012-003939-27). All subjects provided written informed consent. 


\section{D-Transit}

The 3D-Transit system is a novel, ambulatory and minimally invasive method. ${ }^{18}$ It consists of an electronic telemetric capsule for ingestion, a detector with four sensors mounted on the lower part of the abdomen, and dedicated software for real-time and post-experimental visualization and analysis (Fig. 1). The capsules (diameter $8 \mathrm{~mm}$, length $21 \mathrm{~mm}$, and density $1.6 \mathrm{~g} / \mathrm{cm}^{3}$ ) contain an electromagnet and a battery allowing approximately 60 hours of recording at $10 \mathrm{~Hz}$ sampling rate or 120 hours of recording at $5 \mathrm{~Hz}$ sampling rate.

When the capsule is ingested, the sensors register the electromagnetic fields of the capsule and convert them into position coordinates $\mathrm{x}, \mathrm{y}$, and $\mathrm{z}$ and orientation angles $\Phi$ and $\theta$ with respect to the detector. Movements and rotation of the capsule are depicted as graphs via the dedicated software (Fig. 2). Thereby, changes in position and orientation of the capsule, reflecting gut contractile activity and progression dynamics, can be studied with respect to anatomical information. The movements can be monitored in real time via Bluetooth communication and all data are continuously stored on a memory card in the detector for later analysis. Artefacts due to respiration and posture change are recorded using a respiration belt and an accelerometer.

\section{Radiopaque Markers}

Total GI transit times were assessed with a standard radiopaque marker method and were compared with data obtained with 3D-Transit. Ten radiopaque markers were ingested on six consecutive days and an abdominal x-ray was taken on day seven. ${ }^{19}$

\section{Assessment of Symptoms}

Diarrhea and other carcinoid symptoms were verified with a one week diary just before investigations. Blood analysis for chromogranin A and serotonin as well as 24 hours urine 5-hydroxyindoleacetic acid (U5-HIAA) were collected during the investigation.

\section{Protocol}

The subjects arrived at the department in the morning after an overnight fast. A standardized meal was served followed by ingestion of the capsule. After intake of the capsule, subjects were allowed to leave the hospital but not to eat for the following 6 hours. All subjects were provided with standardized meals to be cooked and eaten at home for the following 3 days or until ex-

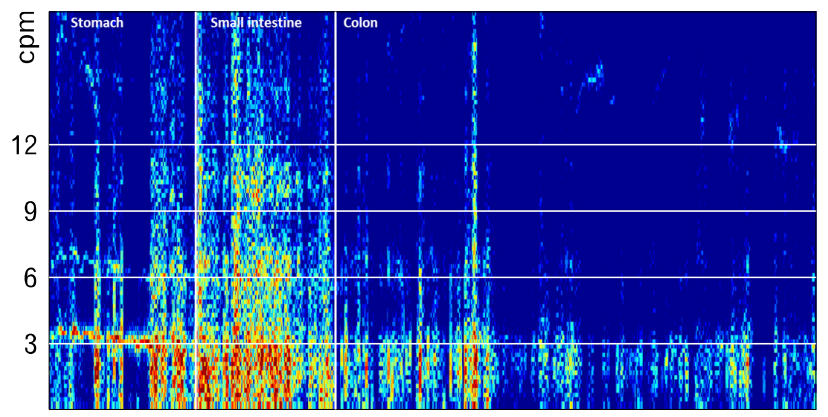

Figure 3. Frequency of contractions. Pyloric and ileocecal passages are determined from changes in gastrointestinal contraction frequency. An increase from 3 to approximately 12 contractions per minute (cpm) marks the pyloric passage while an abrupt decrease in contraction frequency to around 3 contractions per minute marks the ileocecal passage.

pulsion of the capsule $(2200 \mathrm{kcal} /$ day for women and 2800 $\mathrm{kcal} /$ day for men). They kept a diary for registration of exact times of defecation and intake of meals and they were provided with an extra battery and a battery charger.

\section{Data Analysis}

Total GI transit time was defined as the time between ingestion and expulsion of the capsule. Based on previously validated principles, regional transit times were determined from changes in contraction frequencies observed on the graphs and on the time frequency map (Fig. 2 and 3) and 2D overview of capsule position. ${ }^{20}$ Gastric emptying was defined as the time from ingestion until pyloric passage, which was identified as a switch from the 3 contractions per minute (cpm), typical of the stomach, to the $12 \mathrm{cpm}$, typical of the duodenum, combined with the highly characteristic 2D image of passage through the duodenal arch. Small intestinal transit time was defined as the time between pyloric passage and ileocecal passage. Ileocecal passage was identified as the change in contraction frequency of $6 \mathrm{cpm}$, characteristic of the distal ileum, to the $3 \mathrm{cpm}$, typical of the colon. Identification of ileocecal passage was supported by a swift movement in the lower right quadrant. Total colorectal transit time was defined as the time between ileocecal passage and expulsion of the capsule. Proximal colonic transit time was defined as the time between ileocecal passage and passage of the hepatic flexure, identified on the $2 \mathrm{D}$ plot. The middle/distal colonic transit time was defined as the time between passage of the hepatic flexure and expulsion of the capsule.

With 3D-Transit it is possible to track the capsule through the entire GI tract. In the colorectum we defined pansegmental 
movements as movements covering one or more colorectal segments (the ascending, transverse, descending, and/or rectosigmoid part) in less than 2 minutes. We counted the pansegmental movements manually and registered the number of segments they covered in each subject.

\section{Statistical Methods}

All analyses were performed using STATA IC version 11 (StataCorp LP, Texas, USA). Data were non-parametric and presented as median and range. Correlation analyses were performed by Spearman's rho and illustrated by using a two-way scatter plot. Wilcoxon rank-sum test was used to compare transit times in healthy subjects and NET patients. Data were considered statistically significant at $P<0.05$.

\section{Results}

All patients and healthy subjects went through the 3D-Transit examination without any discomfort or adverse events. The basic contraction pattern with $3 \mathrm{cpm}$ in the stomach, 12 declining to 6 cpm in the small intestine, and around $3 \mathrm{cpm}$ in the colon was seen in all patients with NET and in healthy subjects. The capsules were followed through the entire GI tract in all NET patients while two capsules ran out of battery in 2 out of 15 healthy subjects after 54.47 and 56.18 hours, respectively (sampling rate: 10 $\mathrm{Hz}$ ). The capsules were in the cecum and transverse colon when the batteries expired. In both cases, the capsules were retrieved.

A

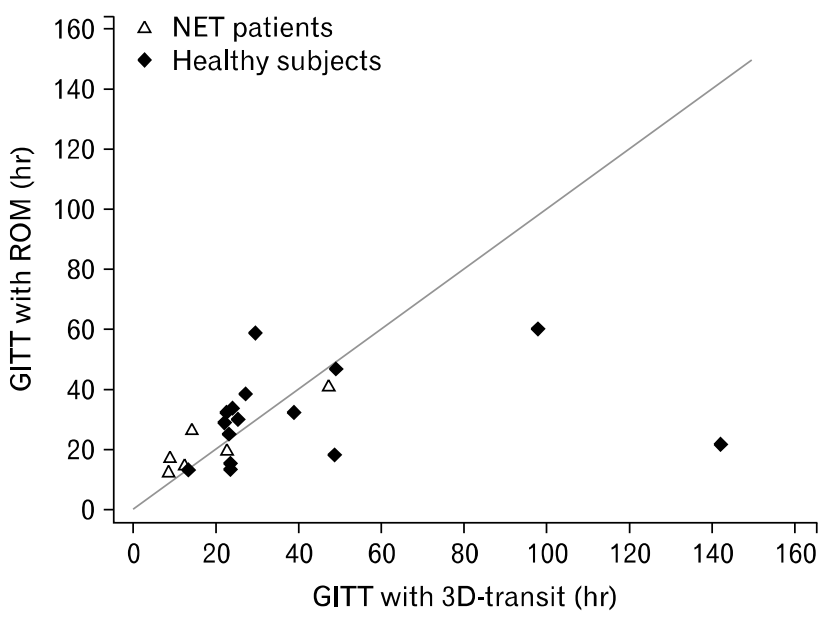

Thus, all regional transit times could be computed for the capsule with battery expiration in the transverse colon. In the case with battery expiration in the cecum, the proximal colonic transit time was defined as the time between entering cecum and end of battery life time. Total colorectal transit time was defined as the time between entering the cecum and retrieving the capsule, while the middle/distal colon transit time was not computed.

\section{Correlation Between 3D-Transit and Radiopaque Markers}

There was a good correlation between GI transit times measured with 3D-Transit and radiopaque markers in patients with NET (Spearman's rho 0.89, $P=0.019$ ) (Fig. 4). The same held true for the combined population of patients and healthy subjects (Spearman's rho 0.64, $P=0.002$ ).

\section{Total and Regional Transit Times in Patients With Neuroendocrine Tumors}

There was no difference in gastric emptying but faster than normal small intestinal transit time (Table 2 and Fig. 5A). The transit through the colorectum was faster in patients with NET than in healthy subjects (Table 2 and Fig. 5B). Patients with NET had significantly faster transit through the proximal colon compared to healthy subjects (Fig. 5C) and there was a trend towards faster than normal transit in the middle/distal colon of patients with NET vs healthy $(P=0.101)$ (Table 2). Patients with NET had faster than normal total GI transit time (Table 2 and

B

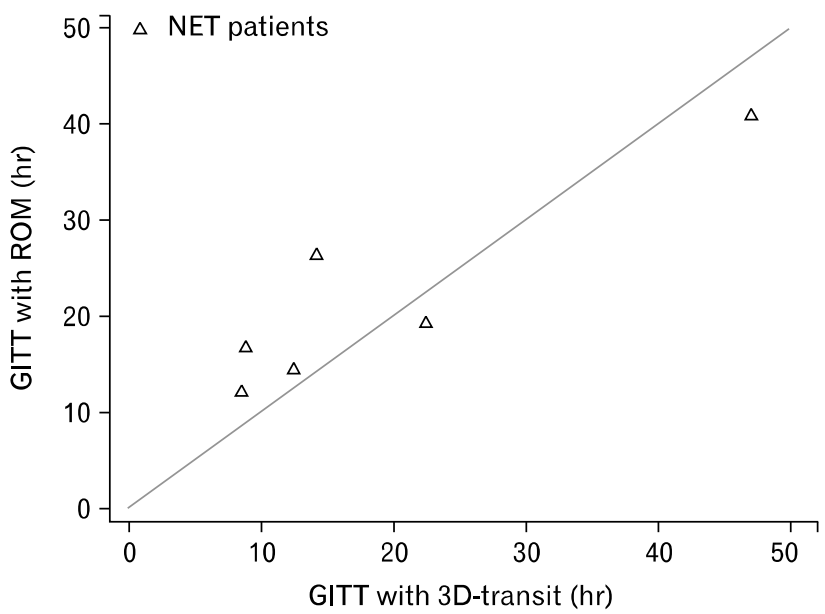

Figure 4. Association between total gastrointestinal transit times (GITT) determined with 3D-Transit and radiopaque markers (ROM). There was a positive correlation between the 2 methods both in the combined group of neuroendocrine tumor (NET) patients and healthy subjects (Spearman's rho $=0.64, P=0.002)(A)$ and in the group of NET patients alone (Spearman's rho $=0.89, P=0.019)(\mathrm{B})$. Lines represent $\mathrm{x}=\mathrm{y}$. Only 6 out of $7 \mathrm{NET}$ patients are depicted as one did not go through the examination with radiopaque markers. 
Tine Gregersen, et al

Table 2. Total and Regional Gastrointestinal Transit Times Measured With 3D-Transit in Healthy Volunteers and Neuroendocrine Tumor Patients With Carcinoid Diarrhea

\begin{tabular}{lccc}
\hline & Healthy subjects & NET patients with diarrhea & $P$-value \\
\hline Gastrointestinal transit time & $25.1(13.1-142.3)$ & $12.5(8.5-47.2)$ & 0.007 \\
Gastric emptying & $3.8(2.1-5.7)$ & $3.8(3.1-4.4)$ & 0.778 \\
Small intestinal transit time & $4.4(1.8-7.2)$ & $3.8(1.4-5.5)$ & 0.044 \\
Proximal colon $^{a}$ & $3.6(0.1-46.3)$ & $0.7(0.02-26.0)$ & 0.045 \\
Middle/distal colon $^{\mathrm{b}}$ & $12.6(0.8-80.4)^{\mathrm{c}}$ & $5.1(1.9-14.1)$ & 0.101 \\
Colorectal transit time & $18.1(5.0-134.0)$ & $5.2(2.9-40.1)$ & 0.012 \\
\hline
\end{tabular}

${ }^{\mathrm{a}}$ Cecum and ascending colon, ${ }^{\mathrm{b}}$ Transverse colon, descending colon and rectosigmoid colon, ${ }^{\mathrm{C}}$ Based on 14 healthy subjects.

NET, neuroendocrine tumor.

Data are given as median (range) in hours.

A

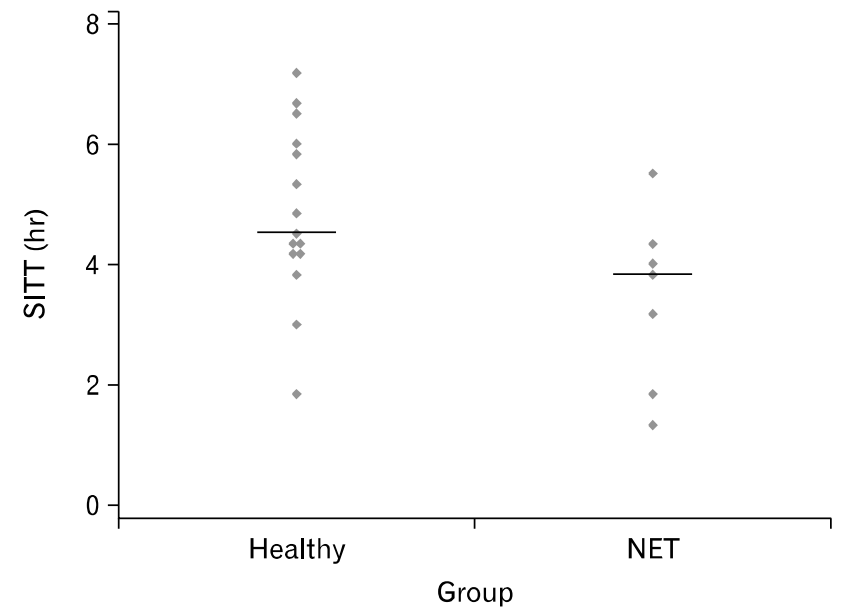

C

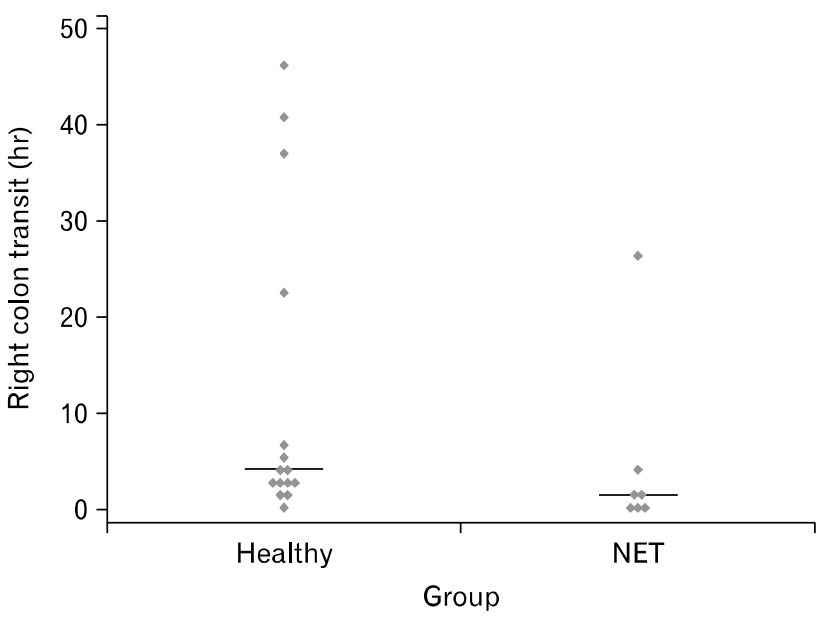

B

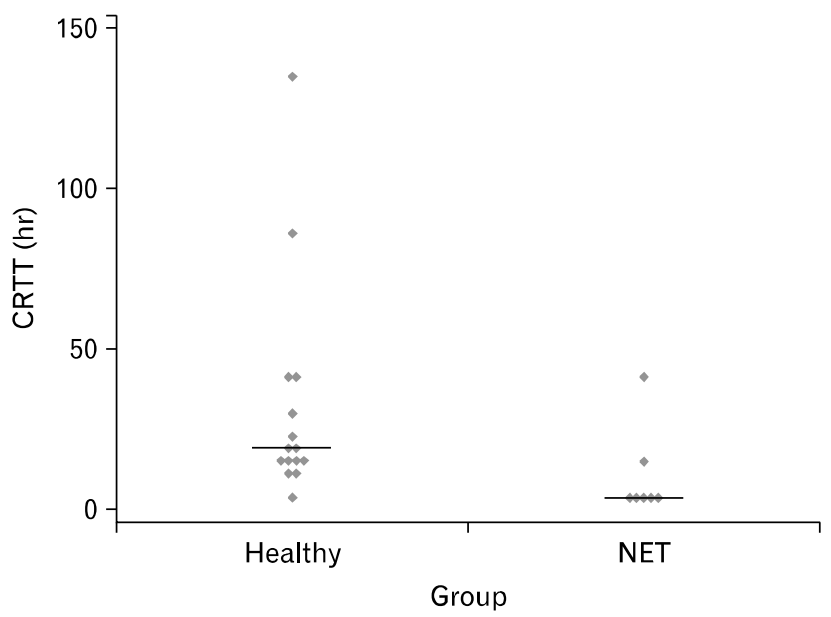

D

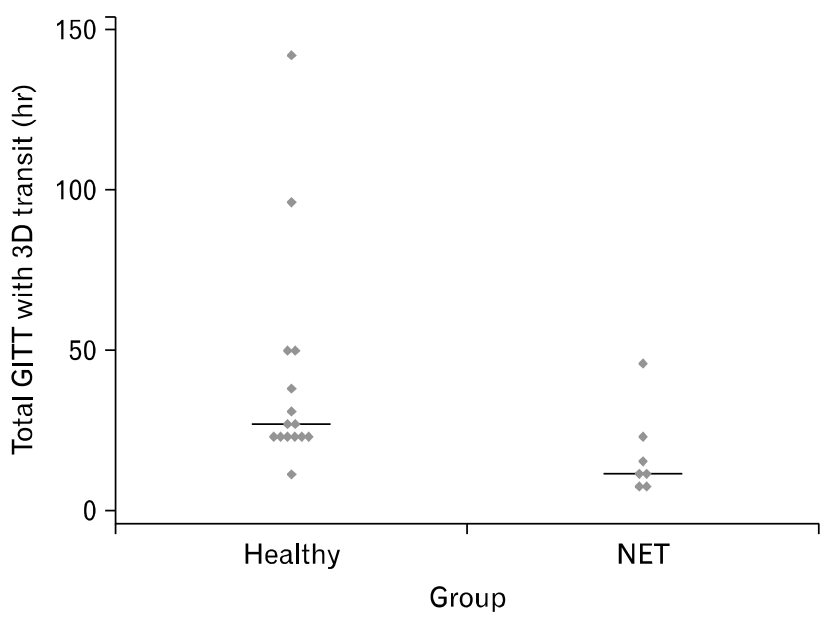

Figure 5. Gastrointestinal transit times in patients with neuroendocrine tumor (NET) and healthy subjects. (A) Small intestinal transit time measured in hours. (B) Colorectal transit time measured in hours. (C) Proximal colonic transit time measured in hours. (D) Total gastrointestinal transit time measured with 3D-Transit in hours. Lines represent the median values. SITT, small intestinal transit time; CRTT, colorectal transit time; GITT, total gastrointestinal transit time. 


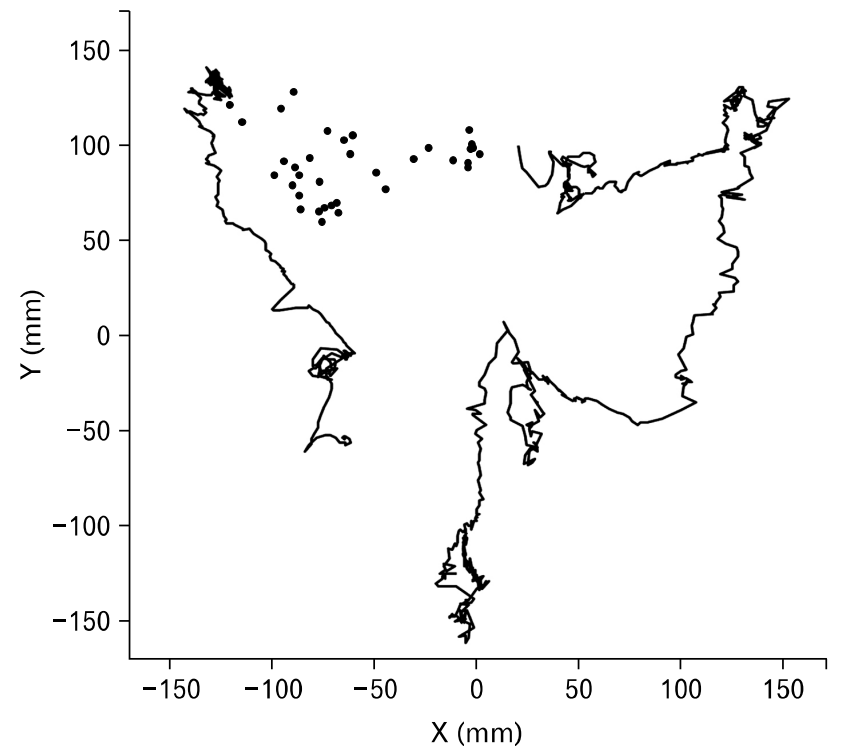

Figure 6. Trajectory of the colonic passage in a healthy subject. The 2 lines illustrate pansegmental colonic movements. The first covers the ascending colon in 22 seconds and the second covers the mid transverse colon, the descending colon and the rectosigmoid colon in 65 seconds. The dots between the 2 pansegmental movements represent slow progression through the hepatic flexure and proximal part of the transverse colon, one dot per 5 minutes.

Fig. 5D). In patients with carcinoid diarrhea, there was an inverse correlation between serotonin levels and total GITT (Spearman's rho $=-0.89, P=0.007$ ). Correlations between total GITT and chromogranin A and U5-HIAA did not reach statistical significance (Spearman's rho $=-0.43, P=0.34$ and Spearman's rho $=-0.71, P=0.11)$.

\section{Pansegmental Colonic Movements}

An example of pansegmental colonic movements is shown in Figure 6. Patients with NET had a median of 2 (range: 1-3) pansegmental colonic movements versus 2 (range: 0-3) in healthy subjects, $(P=0.794)$. Median number of segments covered by pansegmental colonic movements was 3 (range: $1-4)$ in patients with NET and 3 (range $0-4)$ in healthy subjects, $(P=0.912)$. The median number of segments covered by each pansegmental movement was 1 (range: 1-3) in patients with NET and 1.17 (range: $1-2)$ in healthy subjects, $(P=0.685)$. The median frequency of pansegmental colonic movements was 0.45 (range: $0.03-1.02$ ) per hour in patients with NET and 0.07 (range: $0-0.61)$ per hour in healthy subjects $(P=0.045)$. The association between the recorded colorectal transit time and frequency of pansegmental colonic movements is depicted in Figure 7.

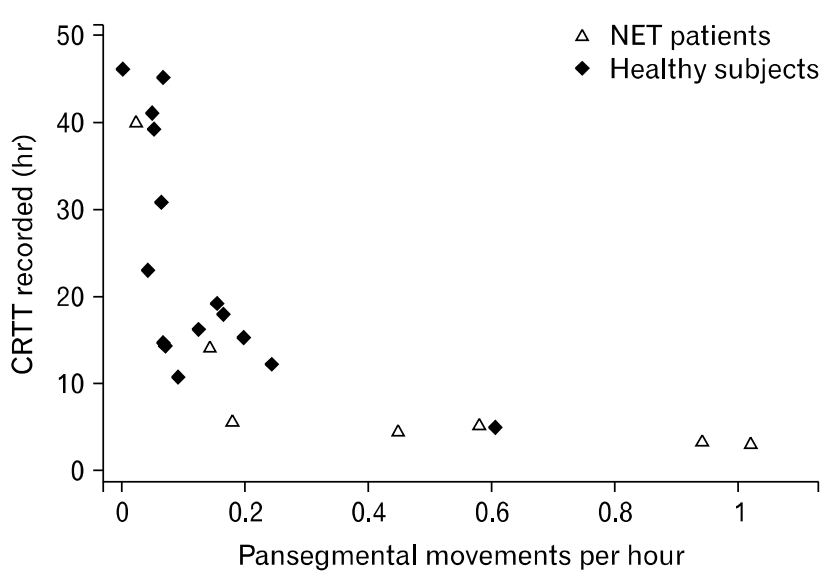

Figure 7. Association between the recorded colorectal transit times (CRTT) and the frequency of pansegmental movements in patients with neuroendocrine tumor (NET) and healthy subjects.

\section{Carcinoid Symptoms}

All patients suffered from diarrhea and reported a median of 6 (range 3-8) bowel movements per day. During the investigation all bowel movements were self-reported in the patients' case report form. It was not possible to compute the exact numbers of bowel movements per day due to fast transit and therefore short investigation time in some patients, but all had more than three per day during the investigation. Three reported flushing and one had asthma like symptoms.

\section{Discussion}

The present study demonstrated that 3D-Transit can be used for assessing regional transit times in patients with diarrhea. Results from 3D-Transit and radiopaque markers were well-correlated and patients with carcinoid diarrhea had significantly faster than normal total and regional GI transit times.

The 3D-Transit system is a promising, novel and minimally invasive method for ambulatory assessment of regional transit times throughout the GI tract. ${ }^{18}$ Furthermore, the system has potential for detailed description of contraction patterns. The recent publication about 3D-Transit concluded that the system is safe, easy-to-use and provides valid data with only minimal data loss in healthy volunteers. ${ }^{18}$ The present study is the first publication applying the 3D-Transit system to patients with chronic diarrhea where transit times are expected to be faster than normal. Use of the 3D-Transit system was well tolerated, basic contraction patterns were easily identified, and regional GI transit times were determined. The method could distinguish between regional 
transit times in patients with the carcinoid syndrome and those in healthy. Total GI transit times obtained with 3D-Transit showed good correlation with those determined with standard radiopaque markers. Thus, the present study supports the use of 3D-Transit in future studies of patients with various forms of diarrhea.

The only other method to allow minimally invasive ambulatory examination of the whole GI tract is the Wireless Motility Capsule (WMC). ${ }^{21}$ The WMC is validated, very easy to use and has been used in several studies. There are, however, fundamental differences between 3D-Transit and WMC. The WMC is based on changes in pressure, temperature and intraluminal $\mathrm{pH}^{22}$ In essence, it allows assessment of whole gut transit time, gastric emptying, small intestinal transit time and total colorectal transit time. In contrast, 3D-Transit is based on the precise anatomical location of one or more electromagnetic capsules during their passage throughout the entire GI tract. ${ }^{18}$ In addition to the information about transit times provided by the WMC, 3D-Transit may allow assessment of regional colonic transit times and, as shown in the present paper, the system holds promise for description of single pansegmental movements within the colon. The data analysis program for 3D-Transit is still in its infancy, but previous studies with MTS-1 allowed description of colonic mass movements, ${ }^{23,24}$ velocity through the different parts of the GI tract, ${ }^{15,20,25-30}$ retrograde movements, ${ }^{23}$ and a gastric motility index.

Our previous studies based on the tracking of a permanent pill were much hampered by the stationary nature of the system, which necessitated the person to sit still in a non-magnetic bed. Thus, previous study protocols were limited to approximately 6 hours. This is not a limitation with the 3D-Transit system. The electromagnet switching on and off with a frequency of $5 \mathrm{~Hz}$ or $10 \mathrm{~Hz}$ makes correction for the influence of other sources of magnetism and, thereby, allows totally ambulatory examinations under near normal circumstances for up to approximately 60 hours at $10 \mathrm{~Hz}$ or twice that at $5 \mathrm{~Hz}$. By changing the phase of the electromagnets, three magnets can be followed simultaneously. If capsules are taken at different time points, simultaneous investigation of movement patterns within different regions of the GI tract is possible. ${ }^{18}$

The carcinoid syndrome is characterized by flushing, diarrhea and, in some patients, asthma like symptoms. Patients with NET suffering from the carcinoid syndrome have an average life time approaching more than 5 years from diagnosis and diarrhea is the single symptom affecting their quality of life the most. ${ }^{1}$ In the present study, we found that patients with the carcinoid syn- drome had significantly faster than normal total GI, small intestinal, proximal colonic and total colorectal transit times. This is in accordance with our earlier findings of normal gastric emptying and faster than normal small intestinal transit times in patients with NET. ${ }^{15}$ However, there is a significant difference between the present and our previous study on GI transit in patients with NET. With 3D-Transit the protocol was not limited to 6 hours. The capsule could be followed throughout the GI tract, there were no missing data due to toilet visit or other breaks and the subjects could live a near normal life in their home environment and at work. All subjects were served standardized meals throughout the study period to minimize confounders. The capsule was ingested after consumption of a standardized meal in order to standardize the motility pattern of the stomach after intake. After ingestion of the capsule all subjects fasted for 6 hours to allow the stomach to enter the fasting state and thus avoid prolonged gastric emptying. Previous studies have shown that the majority of healthy subjects empty capsules from the stomach with a phase III of the migrating myoelectric complex occurring within 6 hours after the last meal. ${ }^{18,31}$ One of the patients in the present study had previously had $26 \mathrm{~cm}$ of the small intestine resected. Diarrhea was present before surgery and we do not consider the minor resection a significant cause of symptoms. Other potential causes of diarrhea in patients with NET include small intestinal bacterial overgrowth, intestinal stenosis with or without mesenteric fibrosis, ischemia, pancreatic insufficiency, bile acid malabsorption, or intestinal failure. Although not systematically ruled out in the present study, none of the patients had known strictures, small intestinal bacterial overgrowth or bile acid diarrhea.

Gastrointestinal transit times in the present study are in accordance with data from von der Ohe et al. who used scintigraphy. ${ }^{17}$ Scintigraphy does, however, require that patients are in hospital during the examination and it involves irradiation.

Transport through the colorectum is mainly caused by mass movements covering large distances of the bowel. The definition of colonic mass movements varies and depends on the techniques used in individual studies. Usually, the velocity should be between $0.7-1.5 \mathrm{~cm} / \mathrm{sec}^{32}$ with a propagation of more than 5-35 $\mathrm{cm}^{33,34}$ The data analysis program available for 3D-Transit only allows valid identification of larger movements. This is likely to be improved with ongoing software development, but in the present study we only registered "pansegmental" movements that carried the capsule through at least one segment. Such movements are mass movements, but be have not registered and included 
mass movements covering less than an entire colorectal segment and some might have passed by the capsule. Interestingly, the frequency of pansegmental movements was significantly higher in patients with NET than in healthy and this is probably the most important pathophysiological mechanism behind faster transit and thereby diarrhea in patients with the carcinoid syndrome. Mass movements mainly occur during the day in association with awakening or in the late postprandial period. ${ }^{35}$ All capsules were ingested in the morning and meals were served at the same time during the investigations. Five out of seven (71\%) patients with NET and 1 out of 15 (7\%) healthy expulsed the capsules during the first day and provided no recordings during the night. This difference may have contributed to the difference in frequency of pansegmental movements.

The clinical presentation of patients with metastatic NET originating in the pancreas usually differs from those with NET originating in the small intestine. Investigating this difference was beyond the scope of the present study, but interestingly, the one patient with pancreatic NET had by far the longest total GI transit and the longest interval between the pansegmental movements of all patients. The patient had 5 bowel movements per day during the investigation and one may speculate whether his symptoms were due to other mechanisms than fast GI transit.

The capsule stayed in the cecum for a minimum of 46 hours in one of the healthy subjects and was expulsed 134 hours after passing the ileocecal valve. This subject had a total GI transit time of 21.6 hours measured by radiopaque markers technique and registered 2 bowel movements per day.

In conclusion, we found that 3D-Transit can be used for safe, ambulatory assessment of regional gastrointestinal transit times and some major colonic contraction patterns in patients with diarrhea. We further conclude that patients with NET and the carcinoid syndrome have faster than normal transit through both the small intestine and the colorectum. The latter is likely to be caused by a high frequency of colonic mass movements.

\section{References}

1. Fröjd C, Larsson G, Lampic C, von Essen L. Health related quality of life and psychosocial function among patients with carcinoid tumours. A longitudinal, prospective, and comparative study. Health Qual Life Outcomes 2007;5:18.

2. Modlin IM, Kidd M, Latich I, Zikusoka MN, Shapiro MD. Current status of gastrointestinal carcinoids. Gastroenterology 2005; 128:1717-1751.

3. Spiller R. Recent advances in understanding the role of serotonin in gastrointestinal motility in functional bowel disorders: alterations in
5-HT signalling and metabolism in human disease. Neurogastroenterol Motil 2007;19(suppl 2):25-31.

4. Hansen MB, Arif F, Gregersen H, Bruusgaard H, Wallin L. Effect of serotonin on small intestinal contractility in healthy volunteers. Physiol Res 2008;57:63-71.

5. Nakajima M, Shiihara Y, Shiba Y, et al. Effect of 5-hydroxytryptamine on gastrointestinal motility in conscious guinea-pigs. Neurogastroenterol Motil 1997;9:205-214.

6. Budhoo MR, Kellum JM. Evidence for a $5-\mathrm{HT}_{4}$ receptor pathway mediating chloride secretion in the rat distal colon. J Surg Res 1994;57:44-48.

7. Lördal M, Hellström PM. Serotonin stimulates migrating myoelectric complex via $5-\mathrm{HT}_{3}$-receptors dependent on cholinergic pathways in rat small intestine. Neurogastroenterol Motil 1999;11:1-10.

8. Tack J, Broekaert D, Corsetti M, Fischler B, Janssens J. Influence of acute serotonin reuptake inhibition on colonic sensorimotor function in man. Aliment Pharmacol Ther 2006;23:265-274.

9. Kvols LK, Moertel CG, O'Connell MJ, Schutt AJ, Rubin J, Hahn RG. Treatment of the malignant carcinoid syndrome. Evaluation of a long-acting somatostatin analogue. N Engl J Med 1986;315:663-666.

10. Janson ET, Oberg K. Long-term management of the carcinoid syndrome. Treatment with octreotide alone and in combination with alpha-interferon. Acta Oncol 1993;32:225-229.

11. O'Toole D, Ducreux M, Bommelaer G, et al. Treatment of carcinoid syndrome: a prospective crossover evaluation of lanreotide versus octreotide in terms of efficacy, patient acceptability, and tolerance. Cancer 2000;88:770-776.

12. Wolin EM. The expanding role of somatostatin analogs in the management of neuroendocrine tumors. Gastrointest Cancer Res 2012;5: 161-168.

13. Janson ET, Sorbye H, Welin S, et al. Nordic guidelines 2014 for diagnosis and treatment of gastroenteropancreatic neuroendocrine neoplasms. Acta Oncol 2014;53:1284-1297.

14. Rinke A, Müller HH, Schade-Brittinger C, et al. Placebo-controlled, double-blind, prospective, randomized study on the effect of Octreotide LAR in the control of tumor growth in patients With metastatic neuroendocrine midgut tumors: a report from the PROMID study group. J Clin Oncol 2009;27:4656-4663.

15. Gregersen T, Grønbæk H, Worsøe J, Schlageter V, Laurberg S, Krogh K. Effects of Sandostatin LAR on gastrointestinal motility in patients with neuroendocrine tumors. Scand J Gastroenterol 2011; 46:895-902.

16. Caplin ME, Pavel M, Ćwikla JB, et al. Lanreotide in metastatic enteropancreatic neuroendocrine tumors. N Engl J Med 2014;371: 224-233.

17. von der Ohe MR, Camilleri M, Kvols LK, Thomforde GM. Motor dysfunction of the small bowel and colon in patients with the carcinoid syndrome and diarrhea. N Engl J Med 1993;329:1073-1078.

18. Haase AM, Gregersen T, Schlageter V, et al. Pilot study trialling a new ambulatory method for the clinical assessment of regional gastrointestinal transit using multiple electromagnetic capsules. Neurogastroenterol Motil 2014;26:1783-1791.

19. Abrahamsson H, Antov S, Bosaeus I. Gastrointestinal and colonic segmental transit time evaluated by a single abdominal $\mathrm{x}$-ray in healthy subjects and constipated patients. Scand J Gastroenterol Suppl 1988;152:72-80. 
20. Worsoe J, Fynne L, Gregersen T, et al. Gastric transit and small intestinal transit time and motility assessed by a magnet tracking system. BMC Gastroenterol 2011;11:145.

21. Maqbool S, Parkman HP, Friedenberg FK. Wireless capsule motility: comparison of the SmartPill GI monitoring system with scintigraphy for measuring whole gut transit. Dig Dis Sci 2009;54: 2167-2174.

22. Lee YY, Erdogan A, Rao SS. How to assess regional and whole gut transit time with wireless motility capsule. J Neurogastroenterol Motil 2014;20:265-270.

23. Hiroz P, Schlageter V, Givel JC, Kucera P. Colonic movements in healthy subjects as monitored by a Magnet Tracking System. Neurogastroenterol Motil 2009;21:838-e57.

24. Stathopoulos E, Schlageter V, Meyrat B, Ribaupierre Y, Kucera P. Magnetic pill tracking: a novel non-invasive tool for investigation of human digestive motility. Neurogastroenterol Motil 2005;17:148-154.

25. Fassov J, Lundby L, Worsøe J, Buntzen S, Laurberg S, Krogh K. A randomised, controlled study of small intestinal motility in patients treated with sacral nerve stimulation for irritable bowel syndrome. BMC Gastroenterol 2014;14:111.

26. Fynne L, Worsøe J, Gregersen T, Schlageter V, Laurberg S, Krogh K. Gastrointestinal transit in patients with systemic sclerosis. Scand J Gastroenterol 2011;46:1187-1193.

27. Hedsund C, Joensson IM, Gregersen T, Fynne L, Schlageter V, Krogh K. Magnet tracking allows assessment of regional gastrointestinal transit times in children. Clin Exp Gastroenterol 2013; 6:201-208.
28. Hedsund C, Gregersen T, Joensson IM, Olesen HV, Krogh K. Gastrointestinal transit times and motility in patients with cystic fibrosis. Scand J Gastroenterol 2012;47:920-926.

29. Karlsen S, Fynne L, Grønbaek H, Krogh K. Small intestinal transit in patients with liver cirrhosis and portal hypertension: a descriptive study. BMC Gastroenterol 2012;12:176.

30. Worsøe J, Fassov J, Schlageter V, Rijkhoff NJ, Laurberg S, Krogh $\mathrm{K}$. Turning off sacral nerve stimulation does not affect gastric and small intestinal motility in patients treated for faecal incontinence. Colorectal Dis 2012;14:e713-e720.

31. Cassilly D, Kantor S, Knight LC, et al. Gastric emptying of a non-digestible solid: assessment with simultaneous SmartPill $\mathrm{pH}$ and pressure capsule, antroduodenal manometry, gastric emptying scintigraphy. Neurogastroenterol Motil 2008;20:311-319.

32. Scott SM. Manometric techniques for the evaluation of colonic motor activity: current status. Neurogastroenterol Motil 2003;15:483-513.

33. Spiller RC, Brown ML, Phillips SF. Decreased fluid tolerance, accelerated transit, and abnormal motility of the human colon induced by oleic acid. Gastroenterology 1986;91:100-107.

34. Herbst F, Kamm MA, Morris GP, Britton K, Woloszko J, Nicholls RJ. Gastrointestinal transit and prolonged ambulatory colonic motility in health and faecal incontinence. Gut 1997;41:381-389.

35. Bassotti G, Iantorno G, Fiorella S, Bustos-Fernandez L, Bilder CR. Colonic motility in man: features in normal subjects and in patients with chronic idiopathic constipation. Am J Gastroenterol 1999;94: 1760-1770. 